\title{
Study on a Kind of Simulation Training System of Photoelectric Theodolite
}

\author{
Changde $\mathrm{Hu}^{1}$, Yongqiang $\mathrm{Li}^{1}$, Huafang Geng ${ }^{1}$ and Jianqiang Liu ${ }^{1}$ \\ ${ }^{1}$ College of Non-commissioned Officer, the Space Engineering University, 102200 Beijing, China
}

\begin{abstract}
A kind of system of target simulation training of photoelectric theodolite is presented. On the basis of the experiment duties, almost 40 operation courses are set according to five modules, which are equipment operation, parameter testing, maintaining, troubleshooting and task flow. Modeling strategy of several kinds of aircraft track is studied and set. The modeling and controlling of the key parts of photoelectric theodolite are achieved with the software of UG and Unity3D. Then the fault waveform of the equipment failure is completed by the soft LabVIEW. With the software Eduis, the multimedia of the operation courses are done. Then scientific and reasonable assessment model is studied and set to evaluate the operation of the trainees. Finally, with the examples of the trainees exams and troops using, it shows the system of target simulation training of photoelectric theodolite meets the requirements.
\end{abstract}

\section{Introduction}

Photoelectric theodolite, as an important photoelectric tracking and measuring equipment, has been widely applied in aviation, aerospace and other military and scientific research fields ${ }^{[1-3]}$. The basic working process of theodolite is that the operator pulls a single rod to make the target enter the television field of view, then the target is captured by a wave gate and then tracked in a closed loop automatically. The key steps are the acquisition and tracking of targets. Existing photoelectric theodolite model training system is mainly used for training the operator's ability to capture and track targets repeatedly. But there is still in blank of the study on the of photoelectric theodolite about composition and working principle, operation method and task flow and fault detection. Based on the requirements of military test and control training, the simulation training system of photoelectric theodolite is studied in this paper. In the course of design, on the basis of meeting the requirements of training subjects and learning from the implementation of virtual reality, focusing on creating realistic training scenarios, make the operator have a strong sense of reality ${ }^{[4-5]}$. It would Fill in the blank of photoelectric theodolite simulation and training examination which is not systematic and comprehensive enough $^{[6-7]}$.

\section{Design and composition of simulation training system}

Simulation training system is composed of simulation computer, simulation training software and display monitor. The simulation training software includes target simulation module, operation training simulation module, measurement and control information feedback simulation module and training assessment module. The system has the functions of simulation training support and assessment and evaluation for actual combat tasks. The trainees could choose the training or assessment items with the training assessment software. In the course of training or assessment, the computer collects the operation information of the trainees, evaluates the operation information received according to certain rules, to finish the assessment of the actual training effect.

\subsection{Composition of simulation training system}

Firstly, real-time background images are captured by VIO card, and then the simulated target position information is obtained from the theoretical trajectory (geocentric coordinates), and the approximate attitude information of the simulated target is obtained after the difference with the theoretical trajectory (geocentric coordinates). Projection matrix data of target surface is got with the operation of the target position information, target attitude information, Target 3D model, theodolite realtime encoder value, theodolite site coordinates, optical system parameters (focus length) and camera parameters (pixel number, pixel size). With unity3D processing to generate the target simulation image, then the background image and the target image are overlapped and fused, the synthesized image is output directly on the display, and the independent synthesized image (PAL or SDI format) output in real time by VIO card. According to the theoretical trajectory information, two-way simulation data are generated, one way of simulation center 
communication data sent to data interactive computer, the other way of simulation mathematics guidance information sent to servo system, which is used to simulate various automatic tracking effects during training. In the assessment mode, after the system recording all the operations, the key operation points of the trainees are compared and graded. The block diagram is as shown in Figure 1.

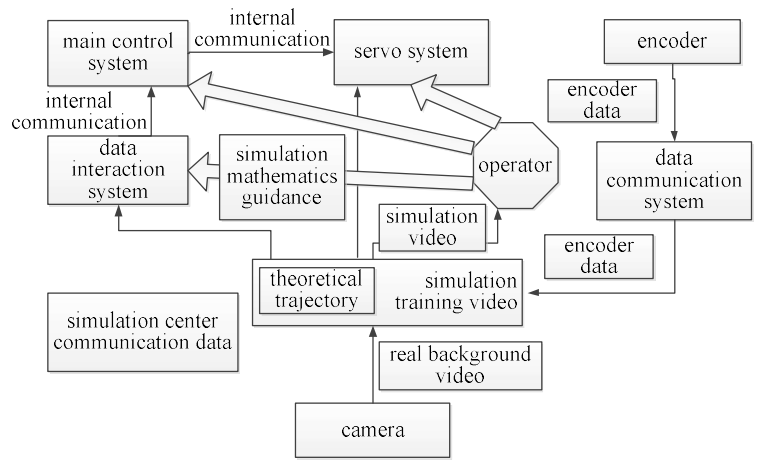

Figure 1. Block diagram of the system

\subsection{Software structure of the photoelectric theodolite simulation training system}

Software architecture system is composed of some form of structured elements, including processing components, data components and connection components. Software architecture system not only determines the organizational structure and topological structure, but also fixes the corresponding relationship between system requirements and components of the system. Layering development is an important architecture with good scalability. And it will not destroy the stability of the original structure when expanding or modifying functions. The software architecture system is divided into three layers by hierarchical structure. They are user interface layer, business processing layer and data storage layer, the relationship between layers is shown in Figure 2.

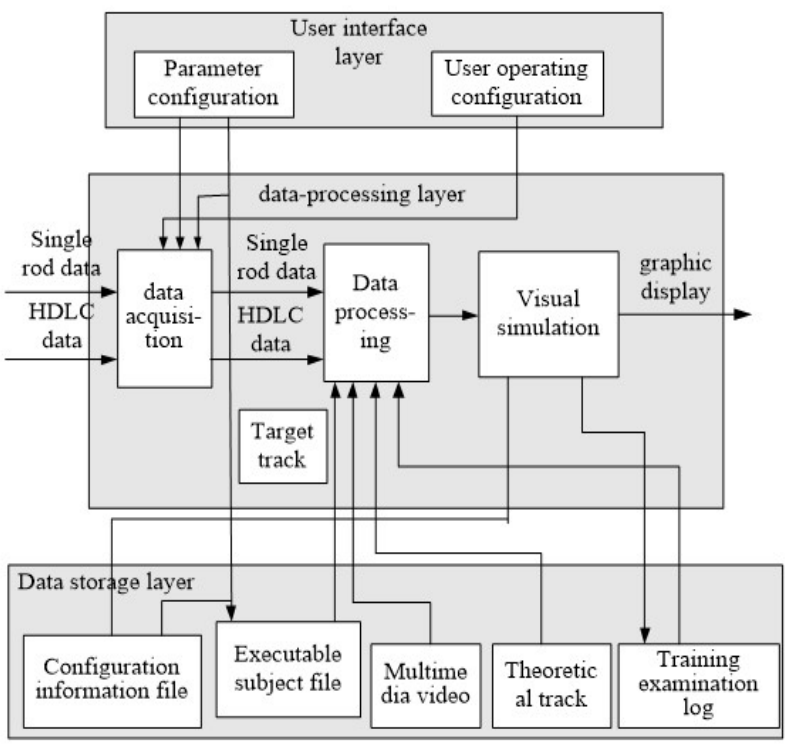

Figure 2. Structural diagram of the system

\subsection{Design of the teaching training assessment design}

The training assessment software is mainly aimed at assessing the training results of various manipulation skills of trainees. The trainees could choose the items to be assessed through training assessment software. Then trainees enter the assessment process, simulate the selected assessment project. During the assessment process, the operation information is collected and recorded. Finally according to certain evaluation rules, evaluate and grading the operation information in real time. The training evaluation subsystem is a system for post-processing analysis of training results. The main indicators of assessment include: the degree of stability, the size of the error, the length of the tracking paragraphs, etc. These indicators need to be considered comprehensively in the evaluation, but also need to take into account the difficulty of ballistic, such as acceleration, speed and so on. The processed data source comes from the hard disk files recorded during the training process. The evaluation results are obtained by comparing the encoder values of the theoretical trajectory with those of the actual training.

\subsubsection{Trajectory difficulty parameter}

The trajectory difficulty parameter is evaluated by equation (1):

$$
\begin{gathered}
A_{\text {dif }}=\left(\left(\sum_{n=0}^{N-1}\left(v_{a n} / v_{a \max }\right)\right) / N+\left(\sum_{n=0}^{N-1}\left(\left|a_{a n} / a_{a \max }\right|\right)\right) / N\right) / 2 \mid \\
E_{\text {dif }}=\left(\left(\sum_{n=0}^{N-1}\left(\mid v_{e n} / v_{e \max }\right)\right) / N+\left(\sum_{n=0}^{N-1}\left(\left|a_{e n} / a_{e \max }\right|\right)\right) / N\right) / 2 \mid \\
\text { Dif }=\left(A_{d i f}+E_{d i f}\right) / 2
\end{gathered}
$$

In the equation (1): Dif - trajectory difficulty, $\in[0$, 1]; $\mathrm{N}$-trajectory point number; $v_{a}$-azimuth velocity; $v_{a \max }$ - maximum azimuth velocity; $v_{e}$ - pitching speed; $v_{e \max }$-maximum pitching speed; $a_{a}$-azimuth acceleration; $a_{a \max }$-maximum azimuth acceleration; $e_{a}$-Pitch acceleration; $e_{a \max }-$ maximum acceleration of pitch.

\subsubsection{Scoring mechanism}

The operation error parameter is evaluated by equation (2):

$$
\begin{aligned}
& \text { OperDiff }=1- \\
& \left(\left(\sum_{m=0}^{N}\left(\left|A r_{m}-A t_{m}\right| / 360\right)\right) / N+\left(\sum_{m=0}^{N}\left(\left|E r_{m}-E t_{m}\right| / 180\right)\right) / N\right) / 2
\end{aligned}
$$

In the equation (2): OperDiff -operational error parameter, $\in[0,1] ; A r_{m}$ - actual training azimuth value; $A t_{m}$ - theoretical azimuth value; $E r_{m}$ — actual training pitch value; $E t_{m}$ - theoretical pitch value. 
The operation error accounts for a weight of 0.5 in the overall score.

The degree of stability is evaluated by equation (3): StatDiff $=1-\left(\left(\sum_{m=0}^{N-1}\left(\left|A_{m+1}-A_{m}\right| / 360\right)\right) / N+\left(\sum_{m=0}^{N-1}\left(\left|E_{m+1}-E_{m}\right| / 180\right)\right) / N\right) / 2$

In the equation (3): StatDiff - stability degree parameter, $\in[0,1], \mathrm{A}$ - actual azimuth value, $\mathrm{E}$-actual pitch value. The stability degree accounts for a weight of 0.2 in the overall score. The tracking length parameter is evaluated by equation (4):

$$
\text { LengDiff }=\operatorname{Tr} / T
$$

In the equation (4): LengDiff - the length of tracking parameter, LengDiff $\in[0,1] ;$ Tr -total target acquisition time; $T$-total time. The degree of comprehensive is evaluated by equation (5):

Score $=($ OperDiff $\times 90) \times 0.5+($ StatDiff $\times 90) \times 0.2+$

$($ LengDiff $\times 90) \times 0.3+$ Dif $\times 10$

In the equation (5): Score-comprehensive scoring, Score $\in[0,100]$.

In the actual use process, according to the different emphasis of the evaluation, the scoring mechanism and algorithm would be properly improved according to the user needs.

\section{Implementation of simulation training system}

\section{1 key technology}

\subsubsection{Establishment of simulation model for moving objects}

1) 3D Flight Target Modeling

3D modeling software is used to build a 3D flying target model, then reading the model data from the 3D graphics data file and drawing the moving target in OpenGL. The 3D model of missile is made by 3DS Studio MAX 8. The built 3DMAX model is saved as Wavefront (.Obj) format. When reading the format file, it generates a data list that includes all the node coordinates, material, texture coordinates, display lists, and so on. Then the model parameters are read and redrawn in OpenGL program. The redrawn model image could reproduce the $3 \mathrm{D}$ model constructed by 3DSMAX software. fitting

2)Two degree interpolation algorithm for flight path

After reading the azimuth and elevation data of the received theodolite, the coordinate could be transformed. The formula (6) is for converting polar coordinates to rectangular coordinates:

$$
\left\{\begin{array}{c}
x=\rho \cos \beta \cos \alpha \\
y=\rho \cos \beta \sin \alpha \\
z=\rho \sin \beta
\end{array}\right.
$$

The track text file contains a series of discrete points on the track (the time interval between points can be set). If the simulated missiles fly on these discrete points, there will be jitter. In order to make the simulation missile more powerful and the flight effect ideal, it is necessary to interpolate the series of points. The 3D coordinates of flight path are interpolated by Lagrange's two degree interpolation algorithm. The interpolated track is as shown in Figure 3.

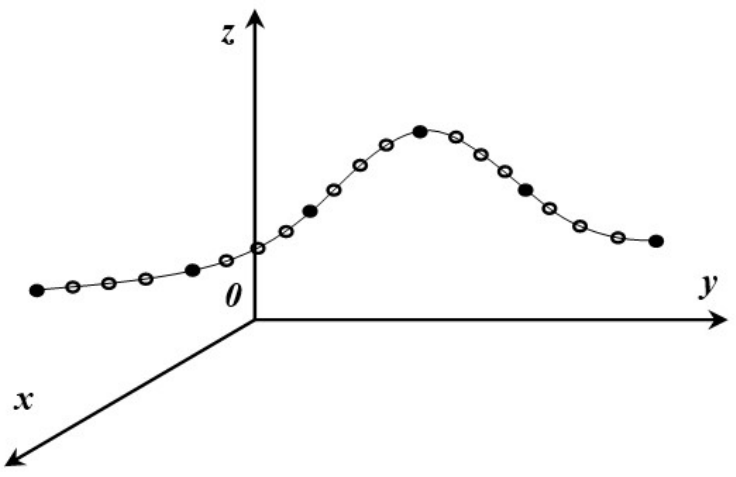

Figure 3. Trajectory diagram with interpolation

The specific algorithm is as follows: constructing the time function of spatial $\mathrm{x}$ coordinates, from track file reading 3 interpolation nodes $x_{k-1}, x_{k}, x_{k+1}$, of which the corresponding moments are $t_{k-1}, t_{k}, t_{k+1}$, then 3 interpolation basic polynomials are built as followings.

$$
\begin{gathered}
l_{k-1}(t)=\frac{\left(t-t_{k}\right)\left(t-t_{k+1}\right)}{\left(t_{k-1}-t_{k}\right)\left(t_{k-1}-t_{k+1}\right)} \\
l_{k}(t)=\frac{\left(t-t_{k}\right)\left(t-t_{k+1}\right)}{\left(t_{k}-t_{k-1}\right)\left(t_{k}-t_{k+1}\right)} \\
l_{k}(t)=\frac{\left(t-t_{k}\right)\left(t-t_{k+1}\right)}{\left(t_{k+1}-t_{k-1}\right)\left(t_{k+1}-t_{k}\right)}
\end{gathered}
$$
(10)

So the interpolation formula is as shown in equation

$$
P(x)=x_{k-1} l_{k-1}(t)+x_{k} l_{k}(t)+x_{k+1} l_{k+1}(t)
$$

The simulation of the flight process is carried out in a cyclic refresh mode. The interpolation point $\mathrm{X}$ coordinate value $P(x)$ can be obtained by taking the time $t$ of flight into the above formula at each refresh. In the same way, the interpolation of $\mathrm{Y}$ and $\mathrm{Z}$ coordinates by the same algorithm can obtain the corresponding interpolation point y coordinate values $\mathrm{P}(\mathrm{y})$ and $\mathrm{Z}$ coordinate values $\mathrm{P}$ (z). In OpenGL, the target's space flight position could be controlled with the function glTranslatef $(\mathrm{x}, \mathrm{y}, \mathrm{z})$.

\subsubsection{Realization of troubleshooting}

The troubleshooting module of this project is achieved based on LabVIEW visual programming software. The 
trainees can select the corresponding test points according to the failure phenomenon when they are trained and assessed, analyzing the cause of failure based on the test waveforms. Then the ability of the trainees to analyze and solve problems could be developed. All the waveforms are generated by LabVIEW.

\subsection{Target simulation training assessment system}

The operation skills of the trainees are developed and obtained by setting up a variety of operation subjects of photoelectric theodolite simulation training assessment system. The operation subjects of the system are mainly based on the main photoelectric equipment. On the basis of the experiment duties, almost 40 operation courses are set according to five modules, which are equipment operation, parameter testing, maintaining, task flow and troubleshooting.

\section{Single rod tracking example}

In the process of photoelectric equipment in the test task, the device operator uses a single rod to capture and track the target. Semi-automatic of single rod tracking is a very important way of tracking. Operators need to improve their operation skills and emergency handling ability through daily tracking training, they can quickly capture and track the tested target stably during the implementation of the task. In order to find out the shortcomings and improve the operation level, each training needs to be evaluated, summarizing the tracking experience. And gradually improve their operational ability.

Single rod semi-automatic tracking requires the operator to capture the target quickly, keeping on tracking the target smoothly, and try to make the target image in the center of the field of view. Therefore, in the process of assessment, these are focused on, the sensitivity of the operator to capture the target, the stability of tracking the target and whether the target is in the center of the field of view.

The simulated trajectory includes key events such as target take-off and program turn. According to the scoring rules, the system gives the final score of 70.3 of a trainee's training situation. The result of the assessment is that the trainees are not stable when the target track changes abruptly. Figure 4 is a tracking chart. The followings are the analyzing.
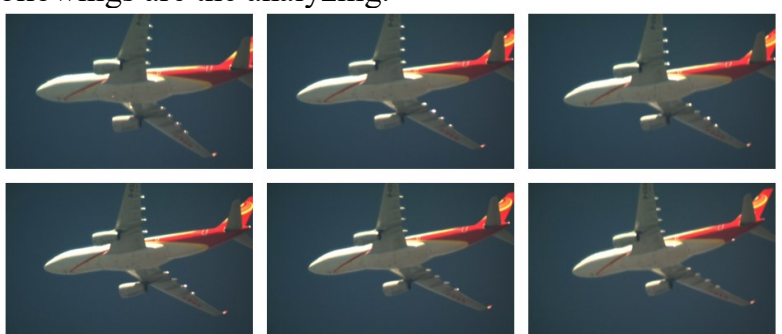

Figure 4. Tracking screen shots

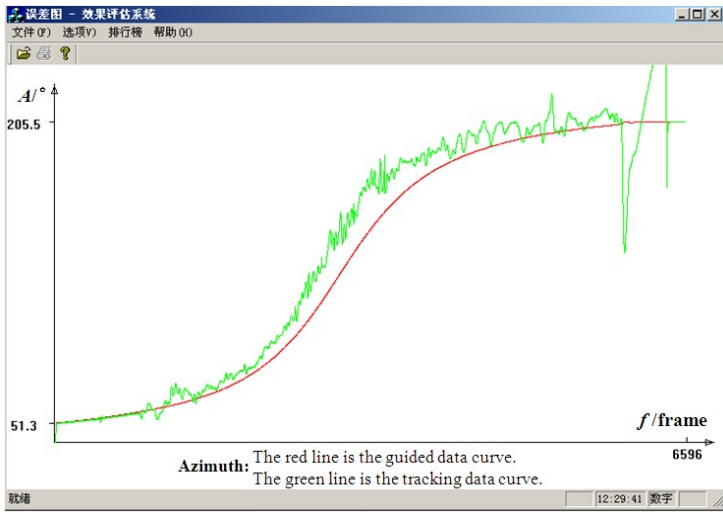

Figure 5. Azimuth tracking error curve

1) Target azimuth tracking analysis

The error curve of tracking aircraft azimuth tracking is shown in Figure 5. The azimuth angle varies greatly with the increase of target velocity. It shows that the miss distance of the target increases gradually, and that the trainees are not stable when the target trajectory changes sharply.

2) Tracking effect evaluation

According to tracking effect, including the stability, tracking time and difficulty of the tracking, the score given by the simulation training assessment system is reasonable. It meets the training level of trainees.

\section{Conclusion}

A photoelectric theodolite simulation training system is designed. Virtual reality technology is used to model targets, backgrounds and trajectories. Considering the motion of the target, including trajectory, velocity and their relationship, the realistic simulation of different types of targets is realized. The modeling and controlling of the key parts of photoelectric theodolite are achieved with the software of UG and Unity3D. Then the fault waveform of the equipment failure is completed by the soft LabVIEW. With the software Eduis, the multimedia of the operation courses are done. Then scientific and reasonable assessment model is studied and set to evaluate the operation of the trainees. Finally, with the examples of the trainees exams and troops using, it shows the system of target simulation training of photoelectric theodolite meets the requirements.

\section{References}

1. Gong Jinglong. Design and implementation of photoelectric theodolite simulation training system[D]. Master's degree paper, Chengdu: Chengdu University of Electronic Science and Technology. 2007.

2. Zheng Huaqiang. The real-time video processing system develop of photoelectric theodolite simulator[D]. Master's degree paper, Chongqing: Chongqing University. 2010.

3. Yan Zhiming, Cheng Bo, Qiu Yanggang. Method study of photoelectric theodolite virtual target 
simulation[J]. Computer Simulation, 2013, 30(2): 383 385.

4. Liu Liang. Simulation training system research and design for a certain type equipment[D]. Master's degree paper, Wuhan: Huazhong University of Science and Technology. 2010.

5. Xiong Shuai. Design and key techniques of virtual reality simulation platform for photoelectric theodolite [D]. Doctor's degree paper, Chengdu: Institute of photoelectric technology, Chinese Academy of Sciences.2013.
6. Wang Xiwen. Study of 3D computer vision for photoelectric theodolite tracking aircraft[D]. Doctor's degree paper, Changchun: Changchun Institute of optics, fine mechanics and physics, Chinese Academy of Sciences, 2010.

7. Xiao Jing. Research and realization of virtual environment simulation of image tracker testing \& evaluation system [D]. Master's degree paper, Xi'an: Xi'an Electronic and Science University, 2009. 